Bull. Mater. Sci., Vol. 22, No. 1, February 1999, pp. 11-13. (C Indian Academy of Sciences.

\title{
High-quality crystal growth and characterization of organic nonlinear optical crystal: 4-dimethylamino-N-methyl-4-stilbazolium tosylate (DAST)
}

\author{
JUNKO YABUZAKI*, YOSHINORI TAKAHASHI, HIROAKI ADACHI, YUSUKE MORI \\ and TAKATOMO SASAKI \\ Department of Electrical Engineering, Osaka University, 2-1 Yamadaoka, Suita, Osaka 565-0871, Japan
}

MS received 23 October 1998

\begin{abstract}
High quality 4-dimethylamino-N-methyl-4-stilbazolium tosylate (DAST) crystal has been successfully grown from saturated methanol solution by a slow cooling method in a Teflon vessel. The full width at half maximum (FWHM) of (001) X-ray diffraction (XRD) rocking curve depends on the growth rate, and the FWHM could be decreased to 15.8 arcsec. Close relation was observed between the mechanical property and crystallinity. The Vickers hardness increased up to 49 on decreasing FWHM.
\end{abstract}

Keywords. DAST; FWHM of XRD rocking curve; Vickers hardness.

\section{Introduction}

Organic nonlinear optical (NLO) materials have been intensely investigated due to their potentially high nonlinearities, and rapid response in electrooptic effect compared to inorganic NLO materials. One of the recently discovered material, 4-dimethylamino- $\mathrm{N}$-methyl4-stilbazolium tosylate (DAST) crystal (Marder et al 1989, 1994; Nakanishi et al 1989), offers large electrooptic coefficient of $r_{111}=77 \pm 8 \mathrm{pm} / \mathrm{V}$ (Pan et al 1996). However, difficulties still remain in crystal growth with sufficient quality and hardness for applications, such as optical and electro-optical (EO) sampling devices. Here we report high quality crystal growth of DAST and its characterization.

\section{Crystal growth}

DAST was synthesized by the condensation of 4-methyl$\mathrm{N}$-methyl pyridinium tosylate, which was prepared from 4-picoline, methyl toluenesulphonate, and 4-N, $\mathrm{N}$-dimethylamino-benzaldehyde in the presence of piperidine. Slow cooling method for crystal growth is usually prepared because of better control on the growth rate, with saturated methanol solution offering relatively bulky crystal morphology as compared to that grown from ethanol or acetone. We have grown bulky DAST crystal to a dimension of $12.2 \times 7.6 \times 5.5 \mathrm{~mm}^{3}$ (Adachi 1998).

Our growth methods can be divided into 2 ways, with and without seeding. We call these 'seed method', and

*Author for correspondence 'spontaneous nucleation method', respectively. In 'seed method', the (001) surface of DAST seed crystal was attached to Teflon rod with silicon bond, and dipped into the saturated methanol solution. The growth rate was varied from $0.14 \mathrm{~mm} /$ day to $1.2 \mathrm{~mm} /$ day by changing cooling rate from 0.04 to $1.2^{\circ} \mathrm{C} /$ day.

On the other hand, in spontaneous nucleation method, the temperature of the DAST solution was decreased below the saturation point until spontaneous nucleation. Ostwald-Miers region was about $5^{\circ} \mathrm{C}$ in our condition. Spontaneously created nucleus was then grown in a cooling rate of about $0.1{ }^{\circ} \mathrm{C} / \mathrm{day}$. Crystals grown by this means had better quality than those grown by seeding method. By this method, DAST crystal as large as $5.1 \times 3.9 \times 1.0 \mathrm{~mm}^{3}$ can be obtained in 10 days (figure 1 ).

\section{Characterization}

Crystallinity of DAST was characterized by XRD in the $\omega$ scan mode. Figure 2 shows $(001)$ peaks of 3 samples grown by the 'seed method'. The relationship between growth rate and crystal quality is indicated. The FWHM of DAST crystals was relatively narrow for crystal grown at slower rate. The best quality of rocking curve 37.4 arcsec was obtained from those grown at a rate of $0.14 \mathrm{~mm} /$ day. In the case of 'spontaneous nucleation method', FWHM of a (001) peak as small as 15.8 arcsec was obtained, which indicated a high crystalline quality of DAST as grown without seed. Mechanical stress induced from the bonding of seed crystal on the Teflon rod seemed to have affected the crystallinity of DAST grown by 'seed method'. 
The X-ray projection topograph (MoK $\alpha$ radiation) of the best crystal grown by spontaneous nucleation method is shown in figure 3. The growth striation and planar defects along $a$ axis are observed. Nevertheless this topograph is relatively high in quality among the organic crystals.

Correlation between the mechanical hardness and the crystallinity was observed. Vickers hardness of DAST

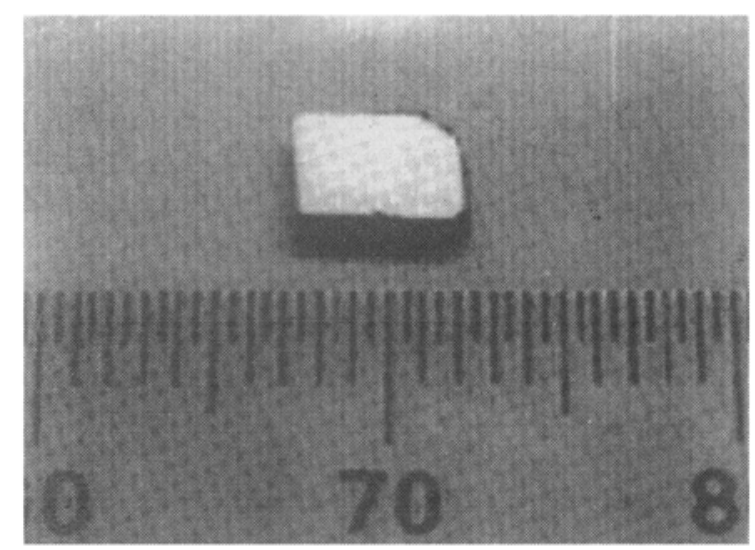

Figure 1. DAST crystal grown by spontaneous nucleation method.

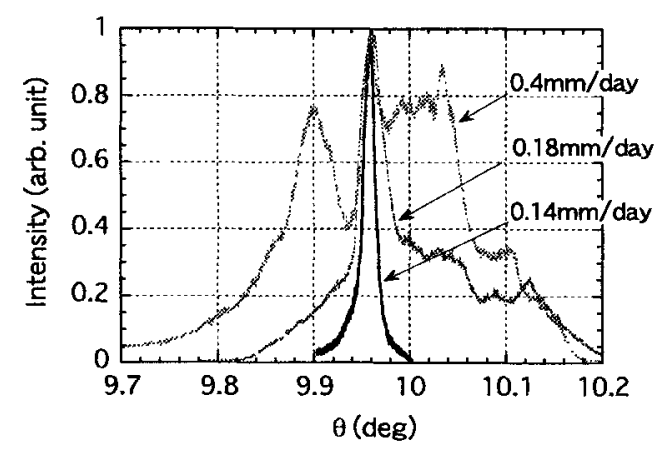

Figure 2. XRD rocking curves of the DAST crystals grown at $0.4 \mathrm{~mm} /$ day (sample 1), $0.18 \mathrm{~mm} /$ day (sample 2), and $0.14 \mathrm{~mm} /$ day (sample 3 ), respectively.

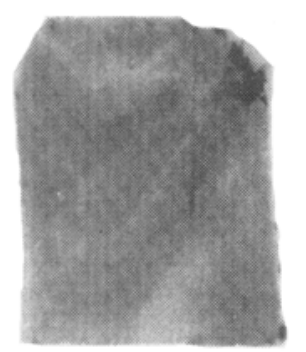

Figure 3. X-ray topograph of DAST crystal grown by spontaneous nucleation $\left(1.0 \mathrm{~mm}\right.$ thick, section $\left.5.1 \times 3.9 \mathrm{~mm}^{2}\right)$. crystals was shown to increase as the rocking curve width (FWHM) decreased (figure 4). A hardness of 49 was obtained from the crystal with rocking curve of 15.8 arcsec FWHM. We think that the orderliness in molecular arrangement between crystal planes of DAST crystals was increased as indicated by the rocking curve. The reduction of plane dislocations resulted in a larger Vickers hardness.

\section{New growing technique 'slope nucleation method'}

Spontaneous nucleation method has shown to be a possible method for growing quality crystals. However, a proper control on the growth condition is necessary, since nuclei tend to combine together and become a polycrystal.

In order to overcome this problem, a new technique called 'slope nucleation method' was investigated (Takahashi et al 1998a). In this method, tiny spontaneous nuclei were grown on a slide as shown in figure 5A. When the gravity of the crystal became larger than the friction of Teflon plate, crystals slided towards the ditch, and they finally stood up and grew at this spot (figure 5B). By this method, we can minimize the contact area between the crystal and Teflon plate, and fix the position of the crystal growth. A dimension of $5.0 \times 4.0 \times 0.5 \mathrm{~mm}^{3}$

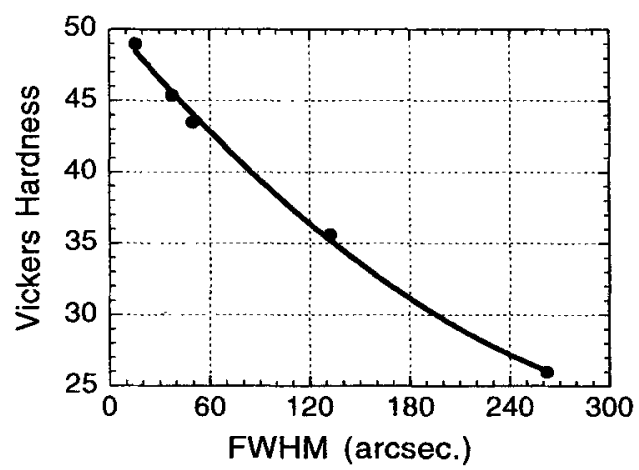

Figure 4. The Vickers hardness of the (001) DAST as a function of FWHM of XRD rocking curve.

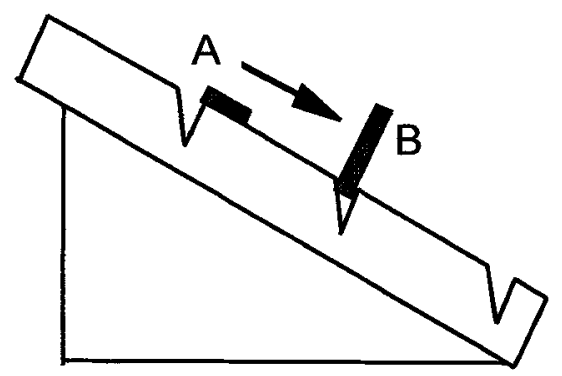

Figure 5. Slope nucleation method. 
was obtained in 5 days. The XRD peak was 25.6 arcsec FWHM which indicated the high crystallinity. With this technique, easier and reliable crystal growth can be obtained.

\section{Conclusion}

High quality DAST crystal can be grown in a Teflon vessel by slow cooling method. XRD analysis showed that the crystallinity of DAST crystal depends on the growth rate. The X-ray topograph of our DAST crystal indicated a relatively high crystallinity. Mechanical hardness increases up to 49 as the FWHM of rocking curve decrease. Controllable crystal growth of DAST by spontaneous nucleation can be obtained with our newly investigated 'slope nucleation method'.

\section{References}

Adachi H 1998 Master Thesis, Osaka University, Japan

Marder S R, Perry J W and Schaefer W P 1989 Science 245 626

Marder S R, Perry J W and Yakymyshyn C P 1994 Chem. Mater. 61137

Nakanishi H, Matsuda H, Okada S and Kato M 1989 Mater. Res. Soc. Int. Mtg. Adv. Mater. 197

Pan F, Knöpfle G, Bosshard Ch, Follonier S, Spreiter R, Wong M S and Günter P 1996 Appl. Phys. Lett. 6913

Takahashi Y, Yabuzaki J, Mori Y and Sasaki T 1998a Abstract 59th Autumn Meet (Japan Society of Applied Physics) $15 \mathrm{p}-\mathrm{ZP}-10$

Takahashi Y, Yabuzaki J, Mori Y and Sasaki T 1998b Int. conf. on organic nonlinear optics p. 80 